\title{
Hemozoin as a novel adjuvant for inactivated whole virion influenza vaccine
}

Ryuta Uraki ${ }^{1}$, Subash C. Das ${ }^{2}$, Masato Hatta ${ }^{2}$, Maki Kiso ${ }^{1}$, Kiyoko Iwatsuki-Horimoto ${ }^{1}$, Makoto Ozawa $^{3,4}$, Cevayir Coban ${ }^{5}$, Ken J Ishii ${ }^{6}$ and Yoshihiro Kawaoka ${ }^{1,2,7,8 *}$

1. Division of Virology, Department of Microbiology and Immunology, Institute of Medical Science, University of Tokyo, Tokyo 108-8639, Japan

2. Influenza Research institute, Department of Pathobiological Sciences, University of Wisconsin-Madison, Madison, WI 53711, USA

3. Laboratory of Animal Hygiene, Joint Faculty of Veterinary Medicine, Kagoshima University, Kagoshima 890-0065, Japan

4. Transboundary Animal Diseases Center, Joint Faculty of Veterinary Medicine, Kagoshima University, Kagoshima 890-0065, Japan

5. Laboratory of Malaria Immunology, Immunology Frontier Research Center(IFReC), Osaka University, Osaka, Japan

6. Laboratory of Adjuvant Innovation, National Institute of Biomedical Innovation; Osaka, Japan; Laboratory of Vaccine Science; IFReC; Osaka University; Osaka, Japan

7. ERATO Infection-Induced Host Responses Project (JST), Saitama 332-0012, Japan

8. Department of Special Pathogens, International Research Center for Infectious Diseases, Institute of Medical Science, University of Tokyo, Minato-ku, Tokyo 108-8639, Japan

Running title: Potential use of synthetic hemozoin as a vaccine adjuvant

Keywords: influenza virus; vaccine; hemozoin; adjuvant; antibody

*Corresponding author's mailing address: Division of Virology, Department of Microbiology and Immunology, Institute of Medical Science, University of Tokyo, 4-6-1, Shirokanedai, Minato-ku, Tokyo, Japan. Phone: +81-3-5449-5504. Fax: +81-3-5449-5408.

E-mail: kawaoka@ims.u-tokyo.ac.jp. 


\section{$37 \quad$ Highlights}

38

39 - Hemozoin enhanced the influenza virus-specific antibody responses in mice.

40 - Mice immunized with hemozoin-adjuvanted inactivated influenza viruses were better protected

41 from lethal influenza virus challenge than were those immunized with inactivated influenza

42 viruses without hemozoin.

43 


\section{Abstract}

Because vaccination is an effective means to protect humans from influenza viruses, extensive efforts have been made to develop not only new vaccines, but also for new adjuvants to

47 enhance the efficacy of existing inactivated vaccines. Here, we examined the adjuvanticity of 48 synthetic hemozoin, a synthetic version of the malarial by-product hemozoin, on the vaccine efficacy

49 of inactivated whole influenza viruses in a mouse model. We found that mice immunized twice with

50 hemozoin-adjuvanted inactivated A/California/04/2009 (H1N1pdm09) or A/Vietnam/1203/2004

51 (H5N1) virus elicited higher virus-specific antibody responses than did mice immunized with

52 non-adjuvanted counterparts. Furthermore, mice immunized with hemozoin-adjuvanted inactivated

53 viruses were better protected from lethal challenge with influenza viruses than were mice immunized

54 with non-adjuvanted inactivated vaccines. Our results show that hemozoin improves the

55 immunogenicity of inactivated influenza viruses, and is thus a promising adjuvant for inactivated 56 whole virion influenza vaccines.

57 
Despite the worldwide surveillance network of influenza viruses, the incidence and

60 prevalence of influenza are hard to predict, as exemplified by the influenza (H1N1) 2009 pandemic

$61[1,2]$. Vaccination stands on the frontlines of influenza infection control: both live attenuated and

62 inactivated influenza vaccines are currently available $[3,4]$. The live attenuated vaccines are more

63 efficient than inactivated vaccines at inducing the mucosal immune responses that play an important

64 role in combating influenza virus infection $[5,6]$. However, because of the safety concerns such as

65 the emergence of revertant and/or reassortant viruses, these live vaccines are licensed in a limited

66 number of countries. By contrast, inactivated vaccines have few safety concerns and are globally

67 available. While they efficiently induce humoral immune responses, a high dose (usually $15 \mu \mathrm{g}$ ) of

68 the inactivated vaccine is required to provide adequate immunity $[7,8]$. Therefore, there is room for

69 improvement in the current influenza vaccines.

enhance the immunogenicity of the inactivated vaccines, adjuvants, such as aluminum compounds

72 and salts, have been considered [10]. Adjuvants are defined as immune modulators that are added to

73 inactivated vaccines to boost the immune responses, enable the use of lower amounts of antigens,

74 and thus expand the vaccine supply $[10,11]$. Although most of the inactivated influenza vaccines

75 currently used are injected via the intramuscular or subcutaneous routes, previous studies have

76 shown that intranasal vaccinations induce antibodies more effectively than do intramuscular or

77 subcutaneous vaccinations [12-14]. However, the alum compounds that are generally used as

78 adjuvants for intramuscular administration do not enhance the efficacy of intranasal vaccines;

79 therefore, to improve the efficacy of intranasal vaccines, novel intranasal adjuvants are required [15].

80 Malaria parasites digest hemoglobin in red blood cells, resulting in the production of

81 potentially toxic heme metabolites [16]. To protect themselves from oxidative damage, the parasites

82 polymerize toxic heme enzymatically into a safer insoluble substance, hemozoin [17]. Recently, 
83 hemozoin and a chemically identical synthetic version of hemozoin (called $\beta$-hematin) have been

84 investigated for their potency as novel adjuvants, and the molecular pathway underlying their

85 immunological function has also been studied. Such studies have demonstrated that purified

86 hemozoin is a non-DNA ligand for Toll-like receptor 9 (TLR9) that may activate innate immune cells

87 via TLR9 [18-20]. This latter point has been a subject of debate, however, because the adjuvant

88 effect of synthetic hemozoin is dependent on MyD88 and not TLR9 [21]. Recently, we reported that

89 hemozoin enhances the protective efficacy of a subcutaneously administered influenza HA split

90 vaccine in a ferret model [22].

91 We speculated that synthetic hemozoin (hereafter referred to only as hemozoin) could serve 92 as a novel intranasal adjuvant for the inactivated influenza vaccine. Accordingly, here we evaluated 93 the adjuvanticity of hemozoin on the vaccine efficacy of intranasally administered inactivated whole 94 virion influenza vaccines in a murine lethal infection model. The results indicate that hemozoin is a 95 promising adjuvant for inactivated whole virion influenza vaccines. 


\section{Cells and viruses.}

Human embryonic kidney HEK293T cells were maintained in Dulbecco's modified Eagle

medium (Lonza, Basel, Switzerland) supplemented with 10\% fetal calf serum (Invitrogen, Carlsbad,

102 CA). Madin-Darby canine kidney (MDCK) cells were maintained in minimum essential medium

103 (MEM) (Invitrogen) supplemented with 5\% newborn calf serum (NCS) (Sigma, St. Louis, MO). All 104 cells were maintained in a humidified incubator at $37^{\circ} \mathrm{C}$ in $5 \% \mathrm{CO}_{2}$.

A/California/04/2009 (H1N1; Ca04), which is an early isolate of influenza (H1N1) 2009 pandemic viruses, and mouse-adapted $\mathrm{Ca} 04$ (MACa04) [23] viruses were propagated in MDCK cells as previously described [24]. A/Vietnam/1203/2004 (H5N1; VN1203) virus, a representative strain of highly pathogenic avian influenza viruses, was grown in MDCK cells and in 10-day-old embryonated chicken eggs to use as challenge viruses and as vaccine and ELISA antigens, respectively. All work involving live VN1203 virus was carried out at the ABSL-3 laboratory of the

111 Influenza Research Institute, UW-Madison, following the protocol designed by Institutional Animal 112 Care and Use Committee (IACUC).

\section{Inactivated influenza virus and adjuvant.}

To inactivate MDCK cell-propagated Ca04 virus and egg-propagated VN1203 virus, 116 formalin (final concentration, $0.1 \%$ ) was added to the viruses, which were then incubated at $4^{\circ} \mathrm{C}$ for 1171 week. The inactivated viruses were purified through a $10 \%-50 \%$ sucrose density gradient and 118 resuspended in phosphate-buffered saline (PBS) as described previously [25]. Inactivation of Ca04

119 viruses was confirmed by passaging them twice in MDCK cells and examining their cytopathic 120 effect; inactivation of VN1203 viruses was confirmed by passaging them twice in embryonated 121 chicken eggs followed by hemagglutination assays. 
122 Synthetic hemozoin, was purified from hemin chloride ( $>98 \%$ pure, Fluka) by using the 123 acid-catalyzed method described previously [21] and was re-suspended in endotoxin-free water with 124 no detectable levels of endotoxin. The synthetic hemozoin concentration was calculated in $\mathrm{mM}(1$ $\mathrm{mg}$ of hemozoin in $1 \mathrm{ml}$ of water was equal to $1 \mathrm{mM})$.

Immunization and protection studies.

For the immunization and protection studies with $\mathrm{Ca} 04$ virus, six-week-old female $\mathrm{BALB} / \mathrm{c}$ mice ( $\mathrm{n}=13$ per group) were anesthetized with isoflurane and intranasally administered with $50 \mu 1$ of PBS, $9 \mathrm{mM}$ hemozoin only, inactivated $\mathrm{Ca} 04$ only $\left[5 \times 10^{6}\right.$ plaque-forming unit (PFU), which corresponds to $0.1 \mu \mathrm{g}$ when the total amount of viral protein was measured by using a BCA protein assay (Thermo Scientific)], or inactivated Ca04 adjuvanted with $9 \mathrm{mM}$ hemozoin, twice with a 2-week interval between the immunizations. Three weeks after the final administration, three mice

134 from each group were euthanized for collection of bronchoalveolar lavage fluid (BALF) and nasal 135 washes. The remaining mice ( $\mathrm{n}=10$ per group) were intranasally challenged with 10 -fold $50 \%$ mouse lethal doses $\left(\mathrm{MLD}_{50}\right)$ of MACa04 virus. On days 3 and 6 post-challenge, three mice each were euthanized and their lungs were collected, homogenized with MEM containing $0.3 \%$ BSA, and examined for virus titers by using plaque assays in MDCK cells. The body weight and survival of the remaining challenged mice ( $\mathrm{n}=4$ per group) were monitored daily for 14 days. euthanized for collection of BALF and nasal washes. The remaining mice ( $\mathrm{n}=11$ per group) were challenged with $100 \mathrm{MLD}_{50}$ of VN1203 virus. On days 3 and 6 post-challenge, three mice each were 144 euthanized and their lungs were collected, homogenized with MEM containing 0.3\% BSA, and 145 examined for virus titers by using plaque assays in MDCK cells. The body weight and survival of the 146 remaining challenged mice ( $\mathrm{n}=5$ per group) were monitored daily for 14 days. 


\section{Detection of virus-specific antibodies.}

Virus-specific antibodies in nasal washes, BALF, and serum were detected by using an ELISA as previously described [25-27]. Briefly, 96-well ELISA plate wells were coated with approximately $0.3 \mu \mathrm{g}$ (in $50 \mu \mathrm{l}$ ) of purified Ca04 or VN1203 virus treated with disruption buffer $(0.5$ M Tris- $\mathrm{HCl}$ [pH 8.0], 0.6 M KCl, and 0.5\% Triton X-100) or sarkosyl, respectively. After incubation of the virus-coated plates with the test samples, virus-specific IgA and IgG antibodies in the samples were detected by using anti-mouse $\operatorname{IgA}$ and $\operatorname{IgG}$ goat antibodies conjugated to horseradish peroxidase (Kirkegaard \& Perry Laboratory Inc., Gaithersburg, MD, Rockland), respectively.

\section{Hemagglutination inhibition assay (HI assay).}

To detect HI antibodies against $\mathrm{Ca} 04$ and VN1203, an HI assay was performed as described previously $[28,29]$. Briefly, serum samples were treated with receptor-destroying enzyme (RDE; Denka Seiken Co., Ltd.) by incubating at $37^{\circ} \mathrm{C}$ for $16-18 \mathrm{~h}$ followed by inactivation at $56^{0} \mathrm{C}$ for 30 min. One volume of turkey or horse red blood cells (RBCs) was then added to 20 volumes of serum and the sera were incubated for $1 \mathrm{~h}$ on ice with intermittent mixing. The samples were then centrifuged at $900 \times \mathrm{g}$ for $5 \mathrm{~min}$, and the supernatants were transferred to new tubes for use in the HI assay. Serially diluted sera (2-fold dilutions) were mixed with 4 HA units of virus antigen and incubated with $0.5 \%$ turkey RBCs or $1 \%$ horse RBCs to determine the extent of hemagglutination inhibition.

\section{Statistical analysis.}

Statistically significant differences in the virus-specific titers $(P<0.05$ and $P<0.01)$ and the survival rates of the challenged mice $(P<0.05)$ were assessed by use of a one-way ANOVA followed by a Dunnett's test and Log-rank statistical analysis, respectively. 


\section{Results}

\section{Hemozoin enhances influenza virus-specific antibody responses in mice.}

To examine the effect of hemozoin on antibody responses elicited by immunization with inactivated influenza viruses, we intranasally administered BALB/c mice with hemozoin-adjuvanted inactivated virus (Ca04 or VN1203 virus, $5 \times 10^{6}$ plaque-forming units (PFU), the total amount of viral protein was $0.1 \mu \mathrm{g}$ ) twice with a 2 -week interval between the immunizations. At three or two weeks after the final administration, we examined the antibody responses to the administered $\mathrm{Ca} 04$ or VN1203 virus by using an ELISA to measure the amount of IgG in the serum and IgA in the BALF and nasal washes (Fig.1). Neither IgG nor IgA against Ca04 or VN1203 virus was appreciably detected in any samples from the PBS- or hemozoin-administered mice. Under these conditions, although one mouse immunized with non-adjuvanted inactivated $\mathrm{Ca} 04$ (Fig. 1A upper panel) and one mouse immunized with non-adjuvanted inactivated VN1203 virus (Fig. 1B upper panel) produced virus-specific IgG in the serum at a detectable level, all of the mice immunized with hemozoin-adjuvanted inactivated Ca04 $(n=3)$ or VN1203 $(n=5)$ virus elicited significantly higher levels of virus-specific IgG in the serum. We also examined the functional properties of the elicited antibodies by using hemagglutination inhibition (HI) assays. For both the Ca04 and VN1203 viruses, greater HI titers were obtained after vaccination with hemozoin-adjuvanted inactivated viruses than with non-adjuvanted inactivated viruses (Fig. 1A\&B upper, right panel), although the titer difference for $\mathrm{Ca} 04$ virus between the hemozoin group and the control groups was not statistically significant (Fig. 1A upper, right panel). Of note, although the addition of hemozoin did not enhance IgA production in the nasal washes or BALF of the inactivated $\mathrm{Ca} 04$ virus-immunized mice, some of the mice immunized with the hemozoin-adjuvanted inactivated VN1203 virus did produce high levels of virus-specific IgA in their nasal washes and BALF (Fig. 1B lower panels). Taken together, these results indicate that hemozoin enhanced the immunogenicity of inactivated influenza viruses, 
198 resulting in more efficient production of virus-specific antibodies.

Hemozoin enhances the efficacy of inactivated influenza vaccine against lethal challenge in

mice.

To further assess the adjuvanticity of hemozoin, mice immunized twice with hemozoin-adjuvanted inactivated Ca04 or VN1203 virus were challenged with a lethal dose of MACa04 (10 $\left.\mathrm{MLD}_{50}\right)$ [23] or VN1203 (100 $\mathrm{MLD}_{50}$ ) virus (Fig. 2). In the MACa04 challenge group, although all of the PBS-administered mice and $75 \%$ of the hemozoin-administered or inactivated $\mathrm{Ca} 04$ virus-immunized mice died, all of the mice immunized with hemozoin-adjuvanted inactivated $\mathrm{Ca} 04$ virus survived (Fig. 2A). Intriguingly, no significant difference was found in Ca04 virus titers in the lungs among the mouse groups tested (Table 1). These results suggest that the adjuvanticity of hemozoin was sufficient to protect mice from lethal challenge with MACa04 virus. virus-immunized mice died following the lethal challenge (Fig. 2B). By contrast, $60 \%$ of the mice 212 immunized with hemozoin-adjuvanted inactivated VN1203 virus survived although mice of all groups experienced body weight loss (Fig. 2B). In accordance with the results of the MACa04 virus challenge, the addition of hemozoin to inactivated VN1203 virus immunization did not affect the

215 virus titers in the lungs of VN1203 virus-challenged mice (Table 2). These results suggest that 216 hemozoin enhanced the vaccine efficacy of the inactivated influenza viruses by modulating host 217 responses, but not by directly inhibiting virus replication. Overall, these results suggest that 218 hemozoin is a promising adjuvant for inactivated influenza vaccines. 


\section{Discussion}

Here, we examined the effect of an adjuvant candidate, hemozoin, on the vaccine efficacy of inactivated whole virion influenza vaccines against lethal challenge in a mouse model. Significantly better virus-specific antibody responses were induced by hemozoin-adjuvanted inactivated virus than by inactivated viruses (Fig. 1). We further demonstrated that the hemozoin-adjuvanted inactivated viruses protected mice from lethal challenges more efficiently than did their non-adjuvanted counterparts with no effect of virus titers in the lungs (Fig. 2, Tables 1 and 2). These results indicate that hemozoin is a promising candidate as an effective adjuvant for inactivated whole virion influenza vaccines.

We observed significantly higher levels of IgA specific for VN1203 virus in the BALF and nasal washes, and of serum IgG, in mice immunized with hemozoin-adjuvanted inactivated VN1203 virus than in mice immunized with non-adjuvanted inactivated VN1203 virus-immunized mice (Fig.1B). These results suggest that hemozoin enhanced the mucosal immune responses and may potentially compensate for the well-recognized weakness of inactivated vaccines [30-32]. By contrast, enhanced IgA production by the hemozoin addition was not observed with the Ca04 virus counterparts (Fig.1A). This contradiction may reflect a difference in immunogenicity between the $\mathrm{Ca} 04$ and VN1203 viruses. Further study is required to clarify the mechanisms by which hemozoin promotes IgA responses after immunization with inactivated vaccines. In addition, hemozoin-adjuvanted inactivated virus protected mice better than non-adjuvanted inactivated viruses although virus titers in lungs were similar between animals immunized with and without the adjuvant (Fig.2, Tables 1 and 2). This finding suggests that hemozoin enhanced the vaccine efficacy of the inactivated influenza viruses by modulating host responses. In the current study, we measured viral loads only in respiratory organs, which are the primary sites of influenza virus replication even for strains that cause systemic infection (e.g., VN1203 virus). A further study to examine the inhibitory effect of hemozoin on systemic spread of influenza viruses may explain the better protection 
afforded by hemozoin-adjuvanted vaccine.

Although hemozoin is a ligand for TLR9 [18-20], studies using TLR9- or MyD88-deficient

247 mice suggest that the potent adjuvant effect of synthetic hemozoin is mediated not via TLR9, but

248 through MyD88 [21]. In addition, previous studies have demonstrated that hemozoin stimulates

249 innate inflammatory responses, inducing neutrophil recruitment via MyD88 [21, 33]. Thus, one of

250 the possible mechanisms underlying the hemozoin-mediated enhanced efficacy of inactivated

251 influenza vaccine may be that hemozoin induces the balanced $T h 1 / T h 2$ responses in a

252 MyD88-dependent manner, leading to the improved immunogenicity of the inactivated influenza

253 viruses and to the better protection against lethal challenge with influenza viruses. Of note, one of

254 four mice administered with only hemozoin survived after the lethal challenge with MACa04 virus

255 (Fig.2A), suggesting that hemozoin itself might have protective effects against influenza virus

256 infection. Additional study is required to clarify the inhibitory effect of hemozoin on influenza virus

257 infection.

258

In conclusion, here, we demonstrated the potential of hemozoin as a novel whole virion influenza vaccine adjuvant. Because the mechanism by which hemozoin enhances immunogenicity remains unclear, we should continue to evaluate the adjuvanticity of hemozoin in the context of influenza vaccination. In addition, to establish the efficacy of hemozoin as an adjuvant, further studies are needed including studies in an additional animal model such as ferrets. 
We thank Dr. Susan Watson for editing the manuscript and Y. Igari and T. Tsukui from 266 Nihon Zenoaq, Co., Ltd for providing synthetic hemozoin. This work was supported, by a 267 Grant-in-Aid for Specially Promoted Research, by the Japan Initiative for the Global Research 268 Network on Infectious Diseases from the Ministry of Education, Culture, Sports, Science, and 269 Technology, Japan, by grants-in-aid from the Ministry of Health, Labour, and Welfare, Japan, by 270 ERATO (Japan Science and Technology Agency), by Strategic Basic Research Programs of Japan 271 Science and Technology Agency, by National Institute of Allergy and Infectious Diseases Public 272 Health Service research grants, and by an NIAID-funded Center for Research on Influenza 273 Pathogenesis (CRIP, HHSN266200700010C). R.U. is supported by JSPS Research Fellowships for 274 young scientists. 


\section{References}

277 [1] Glezen WP. Cold-adapted, live attenuated influenza vaccine. Expert Rev Vaccines 2004 278 Apr;3(2):131-9.

279 [2] Neumann G, Kawaoka Y. The first influenza pandemic of the new millennium. Influenza 280 Other Respir Viruses 2011 May;5(3):157-66.

281 [3] Nabel GJ, Fauci AS. Induction of unnatural immunity: prospects for a broadly protective 282 universal influenza vaccine. Nat Med 2010 Dec;16(12):1389-91.

283 [4] Lambert LC, Fauci AS. Influenza vaccines for the future. N Engl J Med 2010 Nov 284 18;363(21):2036-44.

285 [5] Rimmelzwaan GF, Fouchier RA, Osterhaus AD. Influenza virus-specific cytotoxic T 286 lymphocytes: a correlate of protection and a basis for vaccine development. Curr Opin Biotechnol 2872007 Dec;18(6):529-36.

288 [6] Cox RJ, Brokstad KA, Ogra P. Influenza virus: immunity and vaccination strategies. 289 Comparison of the immune response to inactivated and live, attenuated influenza vaccines. Scand J 290 Immunol 2004 Jan;59(1):1-15.

291 [7] Brokstad KA, Cox RJ, Olofsson J, Jonsson R, Haaheim LR. Parenteral influenza 292 vaccination induces a rapid systemic and local immune response. J Infect Dis 1995 293 Jan;171(1):198-203.

294 [8] Cox RJ, Brokstad KA, Zuckerman MA, Wood JM, Haaheim LR, Oxford JS. An early 295 humoral immune response in peripheral blood following parenteral inactivated influenza vaccination. 296 Vaccine 1994 Aug;12(11):993-9.

297 [9] Tetsutani K, Ishii KJ. Adjuvants in influenza vaccines. Vaccine 2012 Dec 298 14;30(52):7658-61.

299 [10] Reed SG, Orr MT, Fox CB. Key roles of adjuvants in modern vaccines. Nat Med 2013 300 Dec;19(12):1597-608.

301 [11] Dey AK, Srivastava IK. Novel adjuvants and delivery systems for enhancing immune 302 responses induced by immunogens. Expert Rev Vaccines 2011 Feb;10(2):227-51.

303 [12] van Riet E, Ainai A, Suzuki T, Hasegawa H. Mucosal IgA responses in influenza virus 304 infections; thoughts for vaccine design. Vaccine 2012 Aug 31;30(40):5893-900.

305 [13] Tumpey TM, Renshaw M, Clements JD, Katz JM. Mucosal delivery of inactivated influenza 306 vaccine induces B-cell-dependent heterosubtypic cross-protection against lethal influenza A H5N1 307 virus infection. J Virol 2001 Jun;75(11):5141-50.

308 [14] Ainai A, Tamura S, Suzuki T, Ito R, Asanuma H, Tanimoto T, et al. Characterization of 309 neutralizing antibodies in adults after intranasal vaccination with an inactivated influenza vaccine. $\mathrm{J}$ 310 Med Virol 2012 Feb;84(2):336-44.

311 [15] Petrovsky N, Aguilar JC. Vaccine adjuvants: current state and future trends. Immunol Cell 312 Biol 2004 Oct;82(5):488-96. 
313 [16] Francis SE, Sullivan DJ, Jr., Goldberg DE. Hemoglobin metabolism in the malaria parasite 314 Plasmodium falciparum. Annu Rev Microbiol 1997;51:97-123.

315 [17] Arese P, Schwarzer E. Malarial pigment (haemozoin): a very active 'inert' substance. Ann 316 Trop Med Parasitol 1997 Jul;91(5):501-16.

317 [18] Coban C, Ishii KJ, Kawai T, Hemmi H, Sato S, Uematsu S, et al. Toll-like receptor 9

318 mediates innate immune activation by the malaria pigment hemozoin. J Exp Med 2005 Jan 319 3;201(1):19-25.

320 [19] Parroche P, Lauw FN, Goutagny N, Latz E, Monks BG, Visintin A, et al. Malaria hemozoin 321 is immunologically inert but radically enhances innate responses by presenting malaria DNA to 322 Toll-like receptor 9. Proc Natl Acad Sci U S A 2007 Feb 6;104(6):1919-24.

323 [20] Wu X, Gowda NM, Kumar S, Gowda DC. Protein-DNA complex is the exclusive malaria 324 parasite component that activates dendritic cells and triggers innate immune responses. Journal of 325 immunology 2010 Apr 15;184(8):4338-48.

326 [21] Coban C, Igari Y, Yagi M, Reimer T, Koyama S, Aoshi T, et al. Immunogenicity of 327 whole-parasite vaccines against Plasmodium falciparum involves malarial hemozoin and host TLR9. 328 Cell Host Microbe 2010 Jan 21;7(1):50-61.

329 [22] Onishi M, Kitano M, Taniguchi K, Homma T, Kobayashi M, Sato A, et al. Hemozoin is a 330 potent adjuvant for hemagglutinin split vaccine without pyrogenicity in ferrets. Vaccine 2014 May $331 \quad 23 ; 32(25): 3004-9$.

332 [23] Sakabe S, Ozawa M, Takano R, Iwastuki-Horimoto K, Kawaoka Y. Mutations in PA, NP, 333 and HA of a pandemic (H1N1) 2009 influenza virus contribute to its adaptation to mice. Virus Res 3342011 Jun;158(1-2):124-9.

335 [24] Yamada S, Hatta M, Staker BL, Watanabe S, Imai M, Shinya K, et al. Biological and 336 structural characterization of a host-adapting amino acid in influenza virus. PLoS Pathog 337 2010;6(8):e1001034.

338 [25] Uraki R, Kiso M, Iwatsuki-Horimoto K, Fukuyama S, Takashita E, Ozawa M, et al. A novel 339 bivalent vaccine based on a PB2-knockout influenza virus protects mice from pandemic H1N1 and 340 highly pathogenic H5N1 virus challenges. J Virol 2013 Jul;87(14):7874-81.

341 [26] Kida H, Brown LE, Webster RG. Biological activity of monoclonal antibodies to 342 operationally defined antigenic regions on the hemagglutinin molecule of $\mathrm{A} / \mathrm{Seal} / \mathrm{Massachusetts} / 1 / 80$ 343 (H7N7) influenza virus. Virology 1982 Oct 15;122(1):38-47.

344 [27] Das SC, Hatta M, Wilker PR, Myc A, Hamouda T, Neumann G, et al. Nanoemulsion 345 W805EC improves immune responses upon intranasal delivery of an inactivated pandemic H1N1 346 influenza vaccine. Vaccine 2012 Nov 6;30(48):6871-7.

347 [28] Jia N, Wang SX, Liu YX, Zhang PH, Zuo SQ, Lin Z, et al. Increased sensitivity for 348 detecting avian influenza-specific antibodies by a modified hemagglutination inhibition assay using 349 horse erythrocytes. J Virol Methods 2008 Oct;153(1):43-8.

350 [29] Kayali G, Setterquist SF, Capuano AW, Myers KP, Gill JS, Gray GC. Testing human sera for 
351 antibodies against avian influenza viruses: horse $\mathrm{RBC}$ hemagglutination inhibition vs. 352 microneutralization assays. J Clin Virol 2008 Sep;43(1):73-8.

353 [30] Clements ML, Betts RF, Tierney EL, Murphy BR. Serum and nasal wash antibodies 354 associated with resistance to experimental challenge with influenza A wild-type virus. J Clin 355 Microbiol 1986 Jul;24(1):157-60.

356 [31] Tumpey TM, Renshaw M, Clements JD, Katz JM. Mucosal delivery of inactivated influenza 357 vaccine induces B-cell-dependent heterosubtypic cross-protection against lethal influenza A H5N1 358 virus infection. J Virol 2001 Jun;75(11):5141-50.

359 [32] Benton KA, Misplon JA, Lo CY, Brutkiewicz RR, Prasad SA, Epstein SL. Heterosubtypic 360 immunity to influenza A virus in mice lacking IgA, all Ig, NKT cells, or gamma delta T cells. J 361 Immunol 2001 Jun 15;166(12):7437-45.

362 [33] Shio MT, Kassa FA, Bellemare MJ, Olivier M. Innate inflammatory response to the malarial 363 pigment hemozoin. Microbes Infect 2010 Nov;12(12-13):889-99.

364

365

366 


\section{Figure 1. Virus-specific antibody responses in immunized mice.}

369 Virus-specific antibodies were detected by means of ELISA and HI assays with purified Ca04 (A) or 370 VN1203 (B) virus as a viral antigen. IgG antibody titers (upper, left panels) and HI titers (upper, 371 right panels) in serum and IgA antibody titers in the BALF (lower, left panels), and nasal washes 372 (lower, right panels) from mice intranasally mock-immunized with PBS or hemozoin or immunized 373 with non-adjuvanted or hemozoin-adjuvanted inactivated virus were measured. Values represent 374 antibody titers in individual mice $(A: n=3, B: n=5)$. Statistically significant differences $(*: P<0.05$, $375 * *: P<0.01)$ are indicated.

377 Figure 2. Body weight changes and survival of mice challenged with lethal doses of viruses.

378 Mice were mock-immunized with PBS or hemozoin, or immunized with non-adjuvanted or 379 hemozoin-adjuvanted inactivated virus twice with a 2 -week interval in between the immunizations. 380 Three or four weeks after the final immunization, mice were intranasally challenged with $10 \mathrm{MLD}_{50}$ 381 of MACa04 virus (A: $n=4)$ or 100 MLD $_{50}$ of VN1203 virus (B: $\left.n=5\right)$, respectively. Body weight (left panels) and survival (right panels) were monitored for 14 days after challenge. Values are expressed

383 as mean changes in body weight \pm SD (left panels). Statistically significant differences in the 384 survival rate of immunized mice $(*: P<0.05)$ are indicated (A: right panel) 


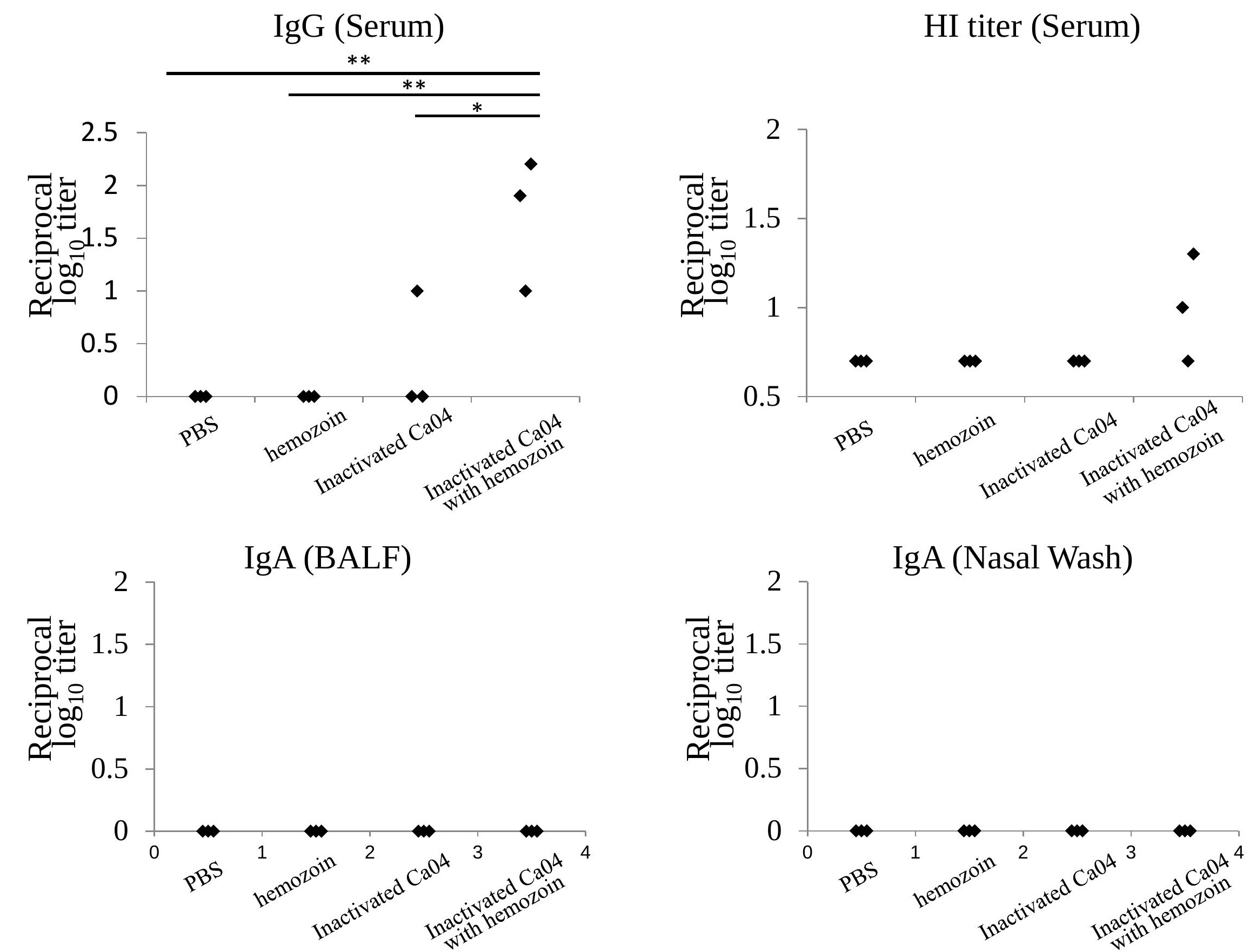


Uraki et al. Figure 1B
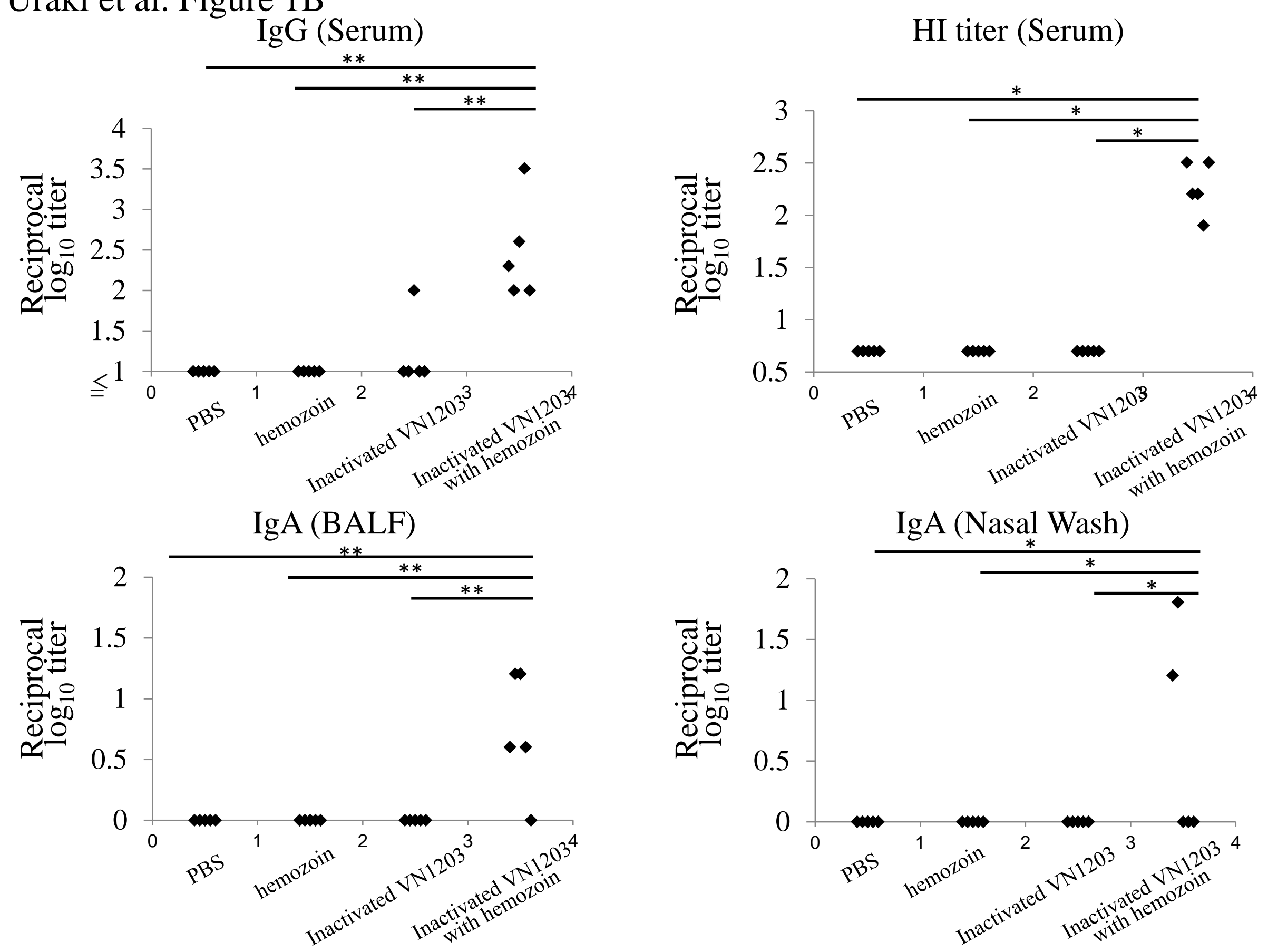
Uraki et al. Figure 2

A $\longrightarrow$ PBS

................ Hemozoin

- - - - Inactivated Ca04 virus (5.0x106 PFU, total viral protein $0.1 \mu \mathrm{g})$

- Inactivated $\mathrm{Ca} 04$ virus $\left(5.0 \times 10^{6} \mathrm{PFU}\right.$, total viral protein $\left.0.1 \mu \mathrm{g}\right)$ with hemozoin
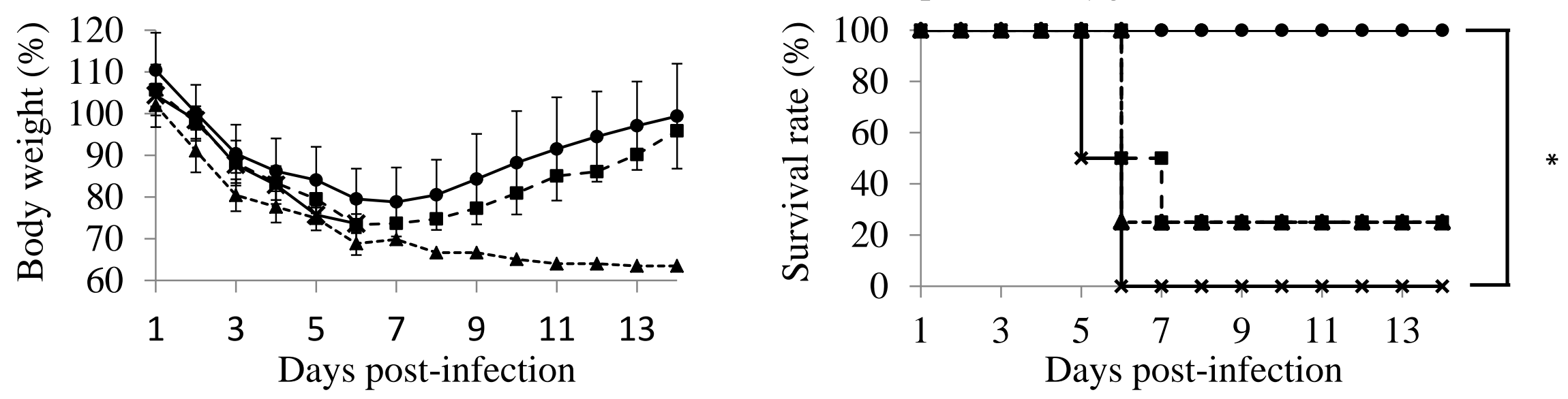

$\mathrm{B} \longrightarrow$ PBS

................ Hemozoin

- - - - Inactivated VN1203 virus (5.0x106 PFU, total viral protein $0.1 \mu \mathrm{g})$

$\longrightarrow$ Inactivated VN1203 virus (5.0x106 PFU, total viral protein $0.1 \mu \mathrm{g})$ with hemozoin
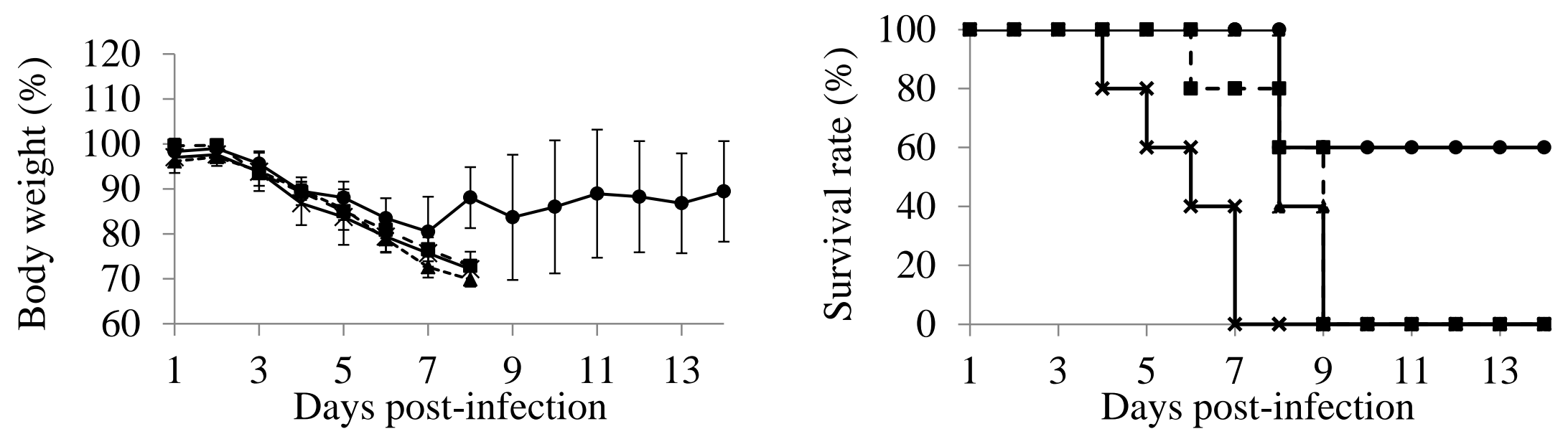